\title{
New Modification of Homotopy Perturbation Method and the Fourth - Order Parabolic Equations with Variable Coefficients
}

\author{
Mohamed Elbadri ${ }^{1,2}$, Tarig. M. Elzaki ${ }^{1,3}$ \\ ${ }^{1}$ Mathematics Department, Faculty of Sciences, Sudan University of Sciences and Technology, Khartoum, Sudan \\ ${ }^{2}$ Mathematics Department, Faculty Mathematics and Computer Science, University of Gezira, Khartoum, Sudan \\ ${ }^{3}$ Mathematics Department, Faculty of Sciences and Arts - Alkamil, University of Jeddah Jeddah, Saudi Arabia
}

\section{Email address:}

badry1982@hotmail.com (M. Elbadri), Tarig.alzaki@gmail.com (T. M. Elzaki)

\section{To cite this article:}

Mohamed Elbadri, Tarig. M. Elzaki. New Modification of Homotopy Perturbation Method and the Fourth - Order Parabolic Equations with Variable Coefficients. Pure and Applied Mathematics Journal. Vol. 4, No. 6, 2015, pp. 242-247. doi: 10.11648/j.pamj.20150406.13

\begin{abstract}
In this paper, the exact solution of the fourth - order parabolic equations with variable coefficients is obtained by using a new homotopy perturbation method (NHPM), theoretical consideration are discussed. Finally, three examples are illustrated to show the validity and applicability of the proposed method.
\end{abstract}

Keywords: New Homotopy Perturbation Method (NHPM), Fourth - Order Parabolic Equations

\section{Introduction}

In this paper, we consider the fourth - order parabolic partial differential equations with the variable coefficients of the form;

$$
\begin{aligned}
& \frac{\partial^{2} u}{\partial t^{2}}+\mu(x, y, z) \frac{\partial^{4} u}{\partial x^{4}}+\lambda(x, y, z) \frac{\partial^{4} u}{\partial y^{4}} \\
& +\eta(x, y, z) \frac{\partial^{4} u}{\partial z^{4}}=g(x, y, z), \\
& a<x, y, z<b, t>0
\end{aligned}
$$

Where, $\mu(x, y, z), \lambda(x, y, z)$ and $\eta(x, y, z)$ are positive, Subject to the initial conditions;

$$
u(x, y, z, 0)=f_{0}(x, y, z), u_{t}(x, y, z, 0)=f_{1}(x, y, z)
$$

And the boundary conditions;

$$
\begin{array}{ll}
u(a, y, z, t)=g_{0}(y, z, t), & u(b, y, z, t)=g_{1}(y, z, t) \\
u(x, a, z, t)=k_{0}(x, z, t), & u(x, b, z, t)=k_{1}(x, z, t) \\
u(x, y, a, t)=h_{0}(x, y, t), & u(x, y, b, t)=h_{1}(x, z, t)
\end{array}
$$

$$
\begin{aligned}
& \frac{\partial^{2} u}{\partial x^{2}}(a, y, z, t)=\tilde{g}_{0}(y, z, t), \\
& \frac{\partial^{2} u}{\partial x^{2}}(b, y, z, t)=\tilde{g}_{0}(y, z, t) \\
& \frac{\partial^{2} u}{\partial y^{2}}(x, a, z, t)=\tilde{k}_{0}(x, z, t), \\
& \frac{\partial^{2} u}{\partial y^{2}}(x, b, z, t)=\tilde{k}_{1}(x, z, t) \\
& \frac{\partial^{2} u}{\partial x^{2}}(a, y, z, t)=\tilde{h}_{0}(x, y, t), \\
& \frac{\partial^{2} u}{\partial x^{2}}(x, y, b, t)=\tilde{h}_{1}(x, y, t)
\end{aligned}
$$

Where the functions $f_{i}, g_{i}, k_{i}, h_{i}, \tilde{g}_{i}, \tilde{k}_{i}, \tilde{h}_{i}, i=0,1$ are continuous functions.it is worth mentioning that the problem equation $(1-3)$ arise in the study of the transverse vibrations problem [1]. numerical computations of the transverse vibrations have been carried out by a number of authors for one dimensional space. the main focus of researchers was to obtain numerical solution by using several techniques, such as explicit and implicit finite - difference schemes [2, 3, 4, 5], Evans [6] who expressed the fourth order equation in two space variables as system of two 
second - order equations to be solved by finite difference method in [7] Khaliq and Twizell solved the variable fourth order parabolic equations by using a family of second order method, Evants etc all [8] investigated the fourth order parabolic equation with constant coefficient by using the AGE method, recently Wazwaz [9, 10] Approached the variable coefficient fourth - order parabolic Equation directly by application Adomian decomposition method. Biazer and Ghazrini [11] applied He's variational iteration method and Deniz Agirseven and Turgut Özis [12] obtained the exact solution of the problem by using homotopy perturbation method. He's homotopy perturbation method [13 - 17] has been used by many researcher in physics and engineering to solve various problem [18 - 22]. In this paper we extend the modification of homotopy perturbation method (NHPM) [23 - 26] to obtain the exact solution of variables coefficients fourth order parabolic equations.

\section{Basic Ideas of the (HPM)}

To solve equation (1) with initial condition (2) by NHPM, we construct the following homotopy:

$$
\begin{gathered}
(1-p)\left(\frac{\partial^{2} v}{\partial t^{2}}-u_{0}\right)+p\left(\frac{\partial^{2} v}{\partial t^{2}}+\mu(x, y, z) \frac{\partial^{2} v}{\partial x^{2}}+\lambda(x, y, z) \frac{\partial^{2} v}{\partial y^{2}}+\right. \\
\left.\eta(x, y, z) \frac{\partial^{2} v}{\partial z^{2}}-g(x, y, z)\right)=0
\end{gathered}
$$

Or:

$$
\begin{aligned}
\frac{\partial^{2} v}{\partial t^{2}} & =u_{0}-p\left(u_{0}+\mu(x, y, z) \frac{\partial^{2} v}{\partial x^{2}}+\lambda(x, y, z) \frac{\partial^{2} v}{\partial y^{2}}\right. \\
& \left.+\eta(x, y, z) \frac{\partial^{2} v}{\partial z^{2}}-g(x, y, z)\right)
\end{aligned}
$$

Applying the inverse operator, $L^{-1}=\int_{0}^{t} \int_{0}^{t}(\circ) d t d t$, to both sides of Eq. (4) we obtain:

$$
\begin{aligned}
& v(x, y, z, t)=v(x, y, z, 0)+t v_{t}(x, y, z, 0)+\int_{0}^{t} \int_{0}^{t} u_{0} d t d t \\
& -p \int_{0}^{t} \int_{0}^{t}\left(u_{0}+\mu(x, y, z) \frac{\partial^{2} v}{\partial x^{2}}+\lambda(x, y, z) \frac{\partial^{2} v}{\partial y^{2}}\right. \\
& \left.+\eta(x, y, z) \frac{\partial^{2} v}{\partial z^{2}}-g(x, y, z)\right) d t d t
\end{aligned}
$$

Where,

$$
v(x, y, z, 0)=f_{0}(x, y, z)
$$

$v_{t}(x, y, z, 0)=f_{1}(x, y, z)$.

Assume the solution of Eq. (4) has the form:

$$
v=v_{0}+p v_{1}+p^{2} v_{2}+\ldots
$$

Suppose the initial approximation to the solution $u_{0}(x, y, z, t)$ has the form:

$$
u_{0}(x, y, z, t)=\sum_{n=0}^{\infty} a_{n}(x, y, z) \rho_{n}(t)
$$

Where, $a_{0}, a_{1}, a_{2}, \ldots$ are unknown coefficients and are specific functions depending on problem. Substituting Eq. (6) into Eq (5) and equating terms of like power $p$, and equating each coefficients of $p$ to zero we get:

$$
\begin{array}{r}
p^{0}: v_{0}(x, y, z, t)=v_{0}(x, y, z, 0)+t v_{0 t}(x, y, z, 0)+\int_{0}^{t} \int_{0}^{t} u_{0} d t d t \\
p^{1}: v_{1}(x, y, z, t)=-\int_{0}^{t} \int_{0}^{t}\left(u_{0}+\mu(x, y, z) \frac{\partial^{4} v_{0}}{\partial x^{4}}+\lambda(x, y, z) \frac{\partial^{4} v_{0}}{\partial y^{4}}\right. \\
+\eta(x, y, z) \frac{\partial^{4} v_{0}}{\left.\partial z^{4}-g(x, y, z)\right) d t d t} \\
p^{2}: v_{2}(x, y, z, t)=-\int_{0}^{t} \int_{0}^{t}\left(\mu(x, y, z) \frac{\partial^{4} v_{1}}{\partial x^{4}}+\lambda(x, y, z) \frac{\partial^{4} v_{1}}{\partial y^{4}}\right. \\
\left.+\eta(x, y, z) \frac{\partial^{4} v_{1}}{\partial z^{4}}\right) d t d t
\end{array}
$$

By solving these equation in such a way that $v_{1}(x, y, z)=0$ then Eq. (7) result in $v_{j}(x, y, z, t)=0, j=1,2,3, \ldots$, therefore the exact solution may be obtained as follows:

$$
\begin{aligned}
u(x, y, z, t) & =v_{0}(x, y, z, t)=f_{0}(x, y, z)+t f_{1}(x, y, z) \\
& +\sum_{n=0}^{\infty} a_{n}(x, y, z) \int_{0}^{t} \int_{0}^{t} \rho_{n}(t) d t d t
\end{aligned}
$$

It is worth mentioning that if $g(x, y, z, t)$ and $u_{0}(x, y, z, t)$ are analytic at $t=t_{0}$ then their Taylor series written as;

$$
\begin{gathered}
u_{0}(x, y, z, t)=\sum_{n=0}^{\infty} a_{n}(x, y, z)\left(t-t_{0}\right)^{n} \\
g(x, y, z, t)=\sum_{n=0}^{\infty} a_{n}^{*}(x, y, z)\left(t-t_{0}\right)^{n}
\end{gathered}
$$

Can be used in Eq. (8), where $a_{0}, a_{1}, a_{2}, \ldots$ are unknown coefficients and $a_{0}^{*}, a_{1}^{*}, a_{2}^{*}, \ldots$ are known ones, which must be computed.

\section{Application}

In this section, we present three examples to illustrate our shames for variable coefficient parabolic equation. 
Example 1.

Consider the one dimensional, variable coefficients fourth order parabolic equation [1];

$$
\frac{\partial^{2} u}{\partial t^{2}}+(1+x) \frac{\partial^{4} u}{\partial x^{4}}=\left(x^{4}+x^{3}-\frac{6}{7 !}\right) \cos t, 0<x<1, t>0
$$

With the initial conditions;

$$
u(x, 0)=\frac{6}{7 !} x^{7}, \frac{\partial u}{\partial t}(x, 0)=0
$$

And the boundary conditions;

$$
\begin{aligned}
& u(0, t)=0 \quad u(1, t)=\frac{6}{7 !} x^{7} \cos t \\
& \frac{\partial^{2} u}{\partial x^{2}}(0, t)=0 \frac{\partial^{2} u}{\partial x^{2}}(1, t)=\frac{1}{20} \cos t
\end{aligned}
$$

To solve Eq. (9) by (NHPM), we construct the following homotopy:

$$
\frac{\partial^{2} v}{\partial t^{2}}=u_{0}-p\left[u_{0}+(1+x) \frac{\partial^{4} v}{\partial x^{4}}-\left(x^{4}+x^{3}-\frac{6}{7 !}\right) \cos t\right]
$$

By integration of Eq. (12) we have:

$$
\begin{gathered}
v(x, t)=v(x, 0)+t v_{0}(x, 0)+\int_{0}^{t} \int_{0}^{t} u_{0} d t d t \\
-p \int_{0}^{t} \int_{0}^{t}\left(u_{0}+(1+x) \frac{\partial^{4} v}{\partial x^{4}}-\left(x^{4}+x^{3}-\frac{6}{7 !}\right) \cos t\right) d t d t
\end{gathered}
$$

Assume the solution of Eq. (13) has the following form:

$$
v=v_{0}+p v_{1}+p^{2} v_{2}+\ldots
$$

Substituting Eq. (14) in to Eq. (13) and equating the coefficients of like powers of $p$, results are:

$$
\begin{gathered}
p^{0}: v_{0}(x, t)=v_{0}(x, 0)+t v_{0 t}(x, 0)+\int_{0}^{t} \int_{0}^{t} u_{0} d t d t \\
p^{1}: v_{1}(x, t)=-\int_{0}^{t} \int_{0}^{t}\left(u_{0}+(1+x) \frac{\partial^{4} v_{0}}{\partial x^{4}}\right. \\
\left.-\left(x^{4}+x^{3}-\frac{6}{7 !}\right) \cos t\right) d t d t \\
p^{2}: v_{2}(x, t)=-\int_{0}^{t} \int_{0}^{t}(1+x) \frac{\partial^{4} v_{1}}{\partial x^{4}} d t d t .
\end{gathered}
$$

Assuming $\quad u_{0}(x, t)=\sum_{n=0}^{\infty} a_{n}(x) t^{n} \quad, \quad v(x, 0)=u(x, 0) \quad$, $v_{t}(x, 0)=u_{t}(x, 0)$ and $v_{1}(x, t)=0$

Then we have:

$$
\begin{aligned}
v_{1}(x, t)= & \left(-\frac{a_{0}}{2}-\frac{1}{2} \frac{6 x^{7}}{7 !}\right) t^{2}+\left(-\frac{a_{1}}{6}\right) t^{3} \\
& +\left(-\frac{a_{0_{x x x x}}}{24}-\frac{a_{0_{x x x x}} x}{24}-\frac{x^{4}}{24}-\frac{x^{3}}{24}-\frac{1}{24} \frac{6 x^{7}}{7 !}-\frac{a_{2}}{12}\right) t^{4} \\
& +\left(-\frac{a_{3_{x x x x}}}{20}\right) t^{5}+\ldots
\end{aligned}
$$

It can be easily to shown that:

$$
a_{0}=-\frac{6}{7 !} x^{7}, a_{1}=0, a_{2}=\frac{1}{2} \frac{6}{7 !} x^{7}, a_{3}=0, . .
$$

Therefore we obtain the solution of Eq. (9);

$$
\begin{aligned}
u(x, t)= & \frac{6 x^{7}}{7 !}+a_{0}(x) \frac{t^{2}}{2}+a_{1}(x) \frac{t^{3}}{6} \\
& +a_{2}(x) \frac{t^{4}}{12}+a_{3}(x) \frac{t^{5}}{20}+\ldots=\frac{6}{7 !} x^{7} \cos t
\end{aligned}
$$

This is an exact solution.

Example 2.

We next consider the fourth - order parabolic equation in two variables;

$$
\begin{aligned}
& \frac{\partial^{2} u}{\partial t^{2}}+2\left(\frac{1}{x^{2}}+\frac{x^{4}}{6 !}\right) \frac{\partial^{4} u}{\partial x^{4}}+2\left(\frac{1}{y^{2}}+\frac{y^{4}}{6 !}\right) \frac{\partial^{4} u}{\partial y^{4}}=0 \\
& \frac{1}{2}<x, y<1, t>0
\end{aligned}
$$

With the initial conditions:

$$
u(x, y, 0)=0, \frac{\partial u}{\partial t}(x, y, 0)=2+\frac{x^{6}}{6 !}+\frac{y^{6}}{6 !},
$$

And the boundary conditions:

$$
\begin{aligned}
& u\left(\frac{1}{2}, y, t\right)=\left(2+\frac{(0.5)^{6}}{6 !}+\frac{y^{6}}{6 !}\right) \sin t \\
& u(1, y, t)=\left(2+\frac{1}{6 !}+\frac{y^{6}}{6 !}\right) \sin t \\
& \frac{\partial^{2} u}{\partial x^{2}}\left(\frac{1}{2}, y, t\right)=\frac{(0.5)^{6}}{24} \sin t \quad, \frac{\partial^{2} u}{\partial x^{2}}(1, y, t)=\frac{1}{24} \sin t \\
& \frac{\partial^{2} u}{\partial x^{2}}\left(x, \frac{1}{2}, t\right)=\frac{(0.5)^{6}}{24} \sin t, \frac{\partial^{2} u}{\partial x^{2}}(x, 1, t)=\frac{1}{24} \sin t
\end{aligned}
$$


To solve Eq. (15) by (NHPM), we construct the following homotopy:

$$
\frac{\partial^{2} v}{\partial t^{2}}=u_{0}-p\left(u_{0}+2\left(\frac{1}{x^{2}}+\frac{x^{4}}{6 !}\right) \frac{\partial^{4} v}{\partial x^{4}}+2\left(\frac{1}{y^{2}}+\frac{y^{4}}{6 !}\right) \frac{\partial^{4} v}{\partial y^{4}}\right)
$$

Applying the inverse operator, $L^{-1}=\int_{0}^{t} \int_{0}^{t}(\circ) d t d t$, to both sides of Eq. (18) we obtain:

$$
\begin{gathered}
v(x, y, t)=v(x, y, 0)+t v_{t}(x, y, 0)+\int_{0}^{t} \int_{0}^{t} u_{0} d t d t \\
-p \int_{0}^{t} \int_{0}^{t}\left(u_{0}+2\left(\frac{1}{x^{2}}+\frac{x^{4}}{6 !}\right) \frac{\partial^{4} v}{\partial x^{4}}+2\left(\frac{1}{y^{2}}+\frac{y^{4}}{6 !}\right) \frac{\partial^{4} v}{\partial y^{4}}\right) d t d t
\end{gathered}
$$

Suppose the solution of Eq. (18) have the form Eq. (14) substituting Eq. (14) into Eq. (19) equating the coefficients of like powers of $p$ we get:

$$
\begin{aligned}
& p^{0}: v_{0}(x, y, t)=v_{0}(x, y, 0)+t v_{0 t}(x, y, 0)+\int_{0}^{t} \int_{0}^{t} u_{0}(x, y, t) d t d t . \\
& p^{1}: v_{1}(x, y, t)=-\int_{0}^{t} \int_{0}^{t}\left(u_{0}+2\left(\frac{1}{x^{2}}+\frac{x^{4}}{6 !}\right) \frac{\partial^{4} v_{0}}{\partial x^{4}}+2\left(\frac{1}{y^{2}}+\frac{y^{4}}{6 !}\right) \frac{\partial^{4} v_{0}}{\partial y^{4}}\right) d t d t . \\
& p^{2}: v_{2}(x, y, t)=-\int_{0}^{t} \int_{0}^{t}\left(2\left(\frac{1}{x^{2}}+\frac{x^{4}}{6 !}\right) \frac{\partial^{4} v_{1}}{\partial x^{4}}+2\left(\frac{1}{y^{2}}+\frac{y^{4}}{6 !}\right) \frac{\partial^{4} v_{1}}{\partial y^{4}}\right) d t d t .
\end{aligned}
$$

Assume $u_{0}(x, y, t)=\sum_{n=0}^{\infty} a_{n}(x, y) t^{n}, v(x, y, 0)=u(x, y, 0)$, $v_{t}(x, y, 0)=u_{t}(x, y, 0)$ and solving the above equation for $v_{1}(x, y, t)$ leads to;

$$
\begin{aligned}
v_{1}(x, t)= & \left(-\frac{a_{0}}{2}\right) t^{2}+\left(-\frac{a_{1}}{6}-\frac{1}{6}\left(2+\frac{x^{6}}{6 !}+\frac{y^{6}}{6 !}\right)\right) t^{3} \\
& +\left(\frac{a_{2}}{12}-\left(\frac{1}{x^{2}}+\frac{x^{4}}{6 !}\right) \frac{a_{0_{x x x x}}}{12}-\left(\frac{1}{y^{2}}+\frac{y^{4}}{6 !}\right) \frac{\left.a_{0_{y y y y}}\right) t^{4}}{12}\right. \\
& +\left(-\frac{a_{5}}{20}-\left(\frac{1}{x^{2}}+\frac{x^{4}}{6 !}\right) \frac{a_{1_{x x x x}}}{120}-\left(\frac{1}{y^{2}}+\frac{y^{4}}{6 !}\right) \frac{a_{0_{y y y}}}{120}\right) t^{5}+\ldots
\end{aligned}
$$

By taking $v_{1}(x, y, t)=0$, coefficients $a_{n}, n=1,2,3, \ldots$ can be determined as the following:

$$
a_{0}=0, a_{1}=-\left(2+\frac{x^{6}}{6 !}+\frac{y^{4}}{6 !}\right), a_{2}=0,
$$

$$
a_{3}=\frac{1}{6}\left(2+\frac{x^{6}}{6 !}+\frac{y^{4}}{6 !}\right), . .
$$

Therefore, an exact solution of the Eq. (15) can be expressed as:

$$
\begin{aligned}
u(x, y, t)= & v_{0}(x, y, t) \\
= & \left(2+\frac{x^{6}}{6 !}+\frac{y^{4}}{6 !}\right) t+a_{0}(x, y) \frac{t^{2}}{2}+ \\
& a_{1}(x, y) \frac{t^{3}}{6}+a_{2}(x, y) \frac{t^{4}}{12}+a_{3}(x, y) \frac{t^{5}}{20}+\ldots \\
= & \left(2+\frac{x^{6}}{6 !}+\frac{y^{4}}{6 !}\right) \sin t
\end{aligned}
$$

Example 3.

We finally consider the following three - dimensional fourth - order parabolic Equation;

$$
\begin{gathered}
\frac{\partial^{2} u}{\partial t^{2}}+\left(\frac{1}{4 ! z}\right) \frac{\partial^{4} u}{\partial x^{4}}+\left(\frac{1}{4 ! x}\right) \frac{\partial^{4} u}{\partial y^{4}}+\left(\frac{1}{4 ! y}\right) \frac{\partial^{4} u}{\partial z^{4}}= \\
-\left[\frac{x}{y}+\frac{y}{z}+\frac{z}{x}-\frac{1}{x^{5}}-\frac{1}{y^{5}}-\frac{1}{z^{5}}\right] \cos t \\
\frac{1}{2}<x, y, z<1, t>0
\end{gathered}
$$

With the initial conditions:

$$
u(x, y, z, 0)=\frac{x}{y}+\frac{y}{z}+\frac{z}{x} \quad, \frac{\partial u}{\partial t}(x, y, z, 0)=0
$$

And the boundary conditions:

$$
\begin{aligned}
u\left(\frac{1}{2}, y, z, t\right) & =\left(\frac{1}{2 y}+\frac{y}{z}+2 z\right) \cos t, \\
u(1, y, z, t) & =\left(\frac{1}{y}+\frac{y}{z}+z\right) \cos t \\
u\left(x, \frac{1}{2}, z, t\right) & =\left(2 x+\frac{1}{z}+\frac{z}{x}\right) \cos t, \\
u(x, 1, z, t) & =\left(x+\frac{1}{z}+\frac{z}{x}\right) \cos t \\
u\left(x, y, \frac{1}{2}, t\right) & =\left(\frac{x}{y}+2 y+\frac{1}{2 x}\right) \cos t \\
u(x, y, 1, t) & =\left(\frac{x}{y}+y+\frac{1}{x}\right) \cos t
\end{aligned}
$$




$$
\begin{aligned}
\frac{\partial u}{\partial x}\left(\frac{1}{2}, y, z, t\right)= & \left(\frac{1}{y}-4 z\right) \cos t, \frac{\partial u}{\partial x}(1, y, z, t)=\left(\frac{1}{y}-z\right) \cos t \\
\frac{\partial u}{\partial y}\left(x, \frac{1}{2}, z, t\right) & =\left(-4 x+\frac{1}{z}\right) \cos t \\
\frac{\partial u}{\partial y}(x, 1, z, t) & =\left(-x+\frac{1}{z}\right) \cos t \\
\frac{\partial u}{\partial z}\left(x, y, \frac{1}{2}, t\right) & =\left(-4 y+\frac{1}{x}\right) \cos t, \\
\frac{\partial u}{\partial z}(x, y, 1, t) & =\left(-y+\frac{1}{x}\right) \cos t
\end{aligned}
$$

To solve Eq. (20) by (NHPM), Eq. (5), in this equation will be in conical form as the following:

$$
\begin{gathered}
v(x, y, z, t)=v(x, y, z, 0)+t v_{t}(x, y, z, 0)+\int_{0}^{t} \int_{0}^{t} u_{0} d t d t \\
-p \int_{0}^{t} \int_{0}^{t}\left(u_{0}+\left(\frac{1}{4 ! z}\right) \frac{\partial^{4} v}{\partial x^{4}}+\left(\frac{1}{4 ! x}\right) \frac{\partial^{4} v}{\partial y^{4}}+\left(\frac{1}{4 ! y}\right) \frac{\partial^{4} v}{\partial z^{4}}\right. \\
\left.+\left[\frac{x}{y}+\frac{y}{z}+\frac{z}{x}-\frac{1}{x^{5}}-\frac{1}{y^{5}}-\frac{1}{z^{5}}\right] \cos t\right) d t d t
\end{gathered}
$$

Suppose the solution of Eq. (23) has the following form:

$$
v=v_{0}+p v_{1}+p^{2} v_{2}+\ldots
$$

Substituting Eq. (24) into Eq. (23) and equating the coefficients of like powers of $p$, to obtain:

$$
\begin{aligned}
p^{0}: v_{0}(x, y, z, t)= & v_{0}(x, y, z, 0)+t v_{0 t}(x, y, z, 0) \\
& +\int_{0}^{t} \int_{0}^{t} u_{0}(x, y, z, t) d t d t . \\
p^{1}: v_{1}(x, y, z, t)= & \int_{00}^{t} \int_{0}^{t}\left(u_{0}+\left(\frac{1}{4 ! z}\right) \frac{\partial^{4} v_{0}}{\partial x^{4}}\right. \\
& +\left(\frac{1}{4 ! x}\right) \frac{\partial^{4} v_{0}}{\partial y^{4}}+\left(\frac{1}{4 ! y}\right) \frac{\partial^{4} v_{0}}{\partial z^{4}} \\
& \left.+\left[\frac{x}{y}+\frac{y}{z}+\frac{z}{x}-\frac{1}{x^{5}}-\frac{1}{y^{5}}-\frac{1}{z^{5}}\right] \cos t\right) d t d t \\
p^{2}: v_{2}(x, y, t)= & -\int_{0}^{t} \int_{0}^{t}\left(\left(\frac{1}{4 ! z}\right) \frac{\partial^{4} v_{1}}{\partial x^{4}}+\left(\frac{1}{4 ! x}\right) \frac{\partial^{4} v_{1}}{\partial y^{4}}+\left(\frac{1}{4 ! y}\right) \frac{\partial^{4} v_{1}}{\partial z^{4}}\right) d t d t
\end{aligned}
$$

By assuming $u_{0}(x, y, z, t)=\sum_{n=0}^{\infty} a_{n}(x, y, z) t^{n}$

$v(x, y, z, 0)=u(x, y, z, 0)$,

$v_{t}(x, y, z, 0)=u_{t}(x, y, z, 0)$, And solving $v_{1}(x, y, t)$, leads to the following result:

$$
\begin{aligned}
v_{1}(x, t)= & \left(-\frac{a_{0}}{2}-\frac{1}{2}\left(\frac{x}{y}+\frac{y}{z}+\frac{z}{x}\right)\right) t^{2} \\
& +\left(-\frac{a_{1}}{6}\right) t^{3}+\left(\frac{a_{2}}{12} \frac{1}{24}\left(\frac{a_{0_{x x x x}}}{4 ! z}+\frac{a_{0_{y y y y}}}{4 ! x}+\frac{a_{0_{z z z z}}}{4 ! y}\right)\right. \\
& \left.+\frac{1}{24}\left(\frac{1}{x^{5}}+\frac{1}{y^{5}}+\frac{1}{z^{5}}\right)-\frac{1}{24}\left(\frac{x}{y}+\frac{y}{z}+\frac{z}{x}\right)\right) t^{4} \\
& +\left(\left(\frac{a}{20} \frac{1}{120}\left(\frac{a_{0_{x x x x}}}{4 ! z}+\frac{a_{0_{y y y y}}}{4 ! x}+\frac{a_{0_{z z z z}}}{4 ! y}\right)\right.\right. \\
-\frac{1}{120}( & \left.\left.\frac{1}{x^{5}}+\frac{1}{y^{5}}+\frac{1}{z^{5}}\right)+\frac{1}{120}\left(\frac{x}{y}+\frac{y}{z}+\frac{z}{x}\right)\right) t^{5}+\ldots
\end{aligned}
$$

By taking $v_{1}(x, y, z, t)=0$, coefficients $a_{n}, n=1,2,3, \ldots$ can be determined as the following:

$$
a_{0}=-\left(\frac{x}{y}+\frac{y}{z}+\frac{z}{x}\right), a_{1}=0, a_{2}=\frac{1}{2}\left(\frac{x}{y}+\frac{y}{z}+\frac{z}{x}\right), a_{3}=0, . .
$$

Therefore, the exact solution of the Eq. (20) can be expressed as:

$$
\begin{aligned}
u(x, y, z, t)= & v_{0}(x, y, z, t) \\
= & \frac{x}{y}+\frac{y}{z}+\frac{z}{x}+a_{0}(x, y, z) \frac{t^{2}}{2}+a_{1}(x, y, z) \frac{t^{3}}{6} \\
& +a_{2}(x, y, z) \frac{t^{4}}{12}+a_{3}(x, y, z) \frac{t^{5}}{20}+\ldots \\
= & \left(\frac{x}{y}+\frac{y}{z}+\frac{z}{x}\right) \cos t .
\end{aligned}
$$

\section{Conclusions}

In this article, NHPM, has been introduced for solving variable coefficients fourth - order parabolic partial differential equations, this method give the exact solution of the problems. This result reveals the method explicit, effective and easy to use.

\section{References}

[1] A. Q. M. Khaliq, E. H. Twizell, A family of second order methods for variable coefficient fourth order parabolic partial differential equations, Internat. J. Comput. Math. 23 (1987) $63-76$.

[2] D. J. Gorman, Free Vibrations Analysis of Beams and Shafts, Wiley, New York, 1975.

[3] C. Andrade, S. McKee, High frequency A. D. I. methods for fourth order parabolic equations with variable coefficients, Internat. J. Comput. Appl. Math 3 (1977) 11-14.

[4] S. D. Conte, A stable implicit difference approximation to a fourth order parabolic equation, J. ACM 4 (1957) 210-212. 
[5] S. D. Conte, W. C. Royster, Convergence of finite difference solution to a solution of the equation of a vibrating beam, Proc. Amer. Math. Soc. 7 (1956) 742-749.

[6] D. J. Evans, A stable explicit method for the finite difference solution of fourth order parabolic partial differential equations, Comput. J. 8 (1965) 280-287.

[7] A. Q. M. Khaliq and E. H. Twizell, A family of second - order methods for variable coefficient fourth - order parabolic partial differential equations, Int. J. Comput. Math 23 (1987), pp. 63-76.

[8] D. J. Evans, W. S. Yousef, A note on solving the fourth order parabolic equation by the AGE method, Internat. J. Comput. Math. 40 (1991) 93-97.

[9] A. M. Wazwaz, Analytic treatment for variable coefficient fourth - order parabolic partial differential equations, Appl. Math. Comput. 123 (2001), pp. 219-227.

[10] A. M. Wazwaz, Exact solutions for variable coefficients fourth - order parabolic partial differential equations in higher - dimensional spaces, Appl. Mathe. Comput. 130 (2002), pp. 415-424.

[11] J. Biazar and H. Ghazvini, He's variational iteration method for fourth - orde parabolic equations, Comput. Math.Appl. 54 (2007), pp. 1047-1054.

[12] Deniz Ağırseven Turgut Öziş, He's homotopy perturbation method for fourth - order parabolic equations,, Int. J. Comput. Math. Jon (2009).

[13] J. H. He, Homotopy perturbation method: a new nonlinear analytical technique, Applied Mathematics and Computation 135 (2003) 73 - 79.

[14] J. H. He, Comparison of Homotopy perturbation method and Homotopy analysis method, Applied Mathematics and Computation 156 (2004) 527 - 539.

[15] J. H. He, The homotopy perturbation method for nonlinear oscillators with discontinuities, Applied Mathematics and Computation 151 (2004) 287 - 292.

[16] J. H. He, Homotopy perturbation method for bifurcation of nonlinear problems, International Journal of Nonlinear Sciences and Numerical Simulation 6 (2005)207 - 208.
[17] J. H. He, Some asymptotic method for strongly nonlinear equations, International Journal of Modern Physics 20 (2006) 1144 - 1199.

[18] J. Biazar, M. Eslami, A new technique for non - linear two dimensional wave equation, Scientia Iranica, Transactions B: Mechanical Engineering, 2013. Mechanics, 35 (1), pp.37 - 43 (2000).

[19] S. T. Mohyud - Din, A. Yildirim, Homotopy perturbation method for advection problems, Nonlinear Science Letters A 1 (2010) 307 - 312.

[20] A. Beléndez, T. Beléndez, A. Markuez, and C. Neipp, Application of He's homotopy perturbation method to conservative truly nonlinear oscillators, Chaos Solitons and Fractals (2006), doi: 10.1016/j.chaos. 2006. 09. 070.

[21] A. Beléndez, T. Beléndez, C. Neipp, A. Hernandez, and M. L. Alvarez, Approximate solutions of a non linear oscillator typified as a mass attached to a stretched elastic wire by the homotopy perturbation method, Chaos Solitons and Fractals (2007): 10.1016/j.chaos, 2007. 01. 089.

[22] A. Belendez, A. Hernandez, T. Belendez, E. Fernandez, M. L. Alvarez, and C. Neip, Application of He's homotopy perturbation method to the Duffing - harmonic oscillator, Int. J. Nonlinear Sci. Numer. Simul. 8 (1) (2007), pp. 79-88.

[23] J. Biazar and M. Eslami, "A new homotopy perturbation method for solving systems of partial differential equations, " Comput. Math Appl., in Press.

[24] J. Biazar and M. Eslami, "A new method for solving the hyperbolic telegraph equation," Comput. Math. Model, in Press.

[25] Mostafa Eslami1 and Jafar Biazar 2, analytical solution of the klein - gordon equation by a new Homotopy Perturbation Method Computational Mathematics and Modeling, Vol. 25, No. 1, March, 2014.

[26] Mohammed ELbadri, A New Homotopy Perturbation Method for Solving Laplace Equation Advances in Theoretical and Applied Mathematics ISSN 0973 - 4554 Volume 8, Number 4 (2013), pp. 237 - 242. 